\title{
Seasonal variation in Guillain-Barré syndrome: a systematic review, meta-analysis and Oxfordshire cohort study
}

\author{
Alastair J S Webb, ${ }^{1}$ Susannah A E Brain, ${ }^{2}$ Ruth Wood, $^{3}$ Simon Rinaldi, ${ }^{1}$ \\ Martin R Turner $^{1}$
}

- Additional material is published online only. To view please visit the journal online (http://dx.doi.org/10.1136/ jnnp-2014-309056).

${ }^{1}$ Nuffield Department of Clinical Neurosciences University of Oxford, Oxford, UK

${ }^{2}$ Addenbrooke's Hospital, University of Cambridge, UK ${ }^{3}$ Clinical Imaging Sciences Centre, Brighton and Sussex Medical School, University of Sussex, UK

Correspondence to Dr Martin R Turner,

Clinical Neurosciences, West Wing Level 3, John Radcliffe Hospital, Oxford OX3 9DU, UK:

martin.turner@ndcn.ox.ac.uk

Received 22 July 2014 Revised 2 October 2014 Accepted 3 December 2014 Published Online First 24 December 2014

\section{CrossMark}

To cite: Webb AJS, Brain SAE, Wood R, et al.

J Neurol Neurosurg

Psychiatry 2015;86:

1196-1201.
ABSTRACT

Introduction Evidence for seasonal variation in incidence and subtype of Guillain-Barré syndrome (GBS) is contradictory, but has implications for provision of neurological services and understanding pathogenesis.

Methods We searched PubMed and EMBASE between inception and January 2014, including all studies reporting seasonal incidence of GBS. We included a retrospective cohort study of patients with GBS at the John Radcliffe Hospital, Oxford 2001-2012 and determined the seasonal variation in GBS incidence and length of stay. The incidence rate ratio (IRR) for winter versus summer was pooled across studies by fixed and random effects meta-analysis weighted by inverse variance, stratified by geographical region, infectious prodrome and GBS subtype.

Results Across 9836 patients from 42 studies there was a $14 \%$ increased risk of GBS in winter versus summer (IRR=1.14, 1.02-1.27, $p=0.020$ ), with significant heterogeneity between studies $\left(I^{2}=77 \%\right.$, $p<0.0001)$, including significant seasonal variation in Oxford ( $n=140 ; p=0.037$ ) for winter versus summer (IRR=1.92, 1.18-3.11, $p=0.004)$ but a non-significantly reduced length of stay for winter versus other seasons ( 15 vs 21 days, $p=0.08$ ). Across all studies, there was greater seasonal variation with respiratory prodrome $(I R R=3.06,1.84-5.11, p<0.0001)$ than diarrhoeal prodrome (IRR=1.10, $0.60-2.00, p=0.76)$ and a greater incidence in winter in Western countries (IRR=1.28), the Far East (IRR=1.20) and Middle East (IRR=1.12), with a lower incidence in the Indian subcontinent (IRR=0.86) and Latin America (IRR=0.75).

Discussion Incidence of GBS was greater in winter than summer, but this was not evident in all geographical regions. This is likely to be related to regional variation in prodromal illnesses.

\section{INTRODUCTION}

Evidence for seasonal variation in Guillain-Barré syndrome (GBS) incidence has been contradictory, with some studies suggesting a winter peak, ${ }^{1-3}$ some studies finding no significant variation ${ }^{45}$ and some reporting summer, spring or autumn peaks. ${ }^{6} 7$ The cause of this heterogeneity has not been systematically assessed, but has been interpreted either as demonstrating no seasonal variation in GBS or demonstrating differences in regional geographical factors. ${ }^{8}$ Studies demonstrating a cluster of cases in the summer months in Northern China ${ }^{9-11}$ supported the hypothesis that rural Chinese populations were more vulnerable to prodromal infections such as Campylobacter jejuni in the summer-rainy season, resulting in acute motor axonal neuropathy (AMAN). In contrast, a number of studies suggested that seasonal variation in GBS was not found in Western countries, ${ }^{45}$ possibly as outbreaks of prodromal infections are less common.

Seasonal variation in GBS may be dependent on seasonality in the precipitating illness, which is thought in some cases to cause cross-reactivity with molecular epitopes on peripheral nerves. ${ }^{12}$ Molecular mimicry has been well-described between GM1 and GD1a gangliosides and lipo-oligosaccharide components of C. jejuni, the commonest identified antecedent infection in AMAN, ${ }^{13}$ while other strains of C. jejuni with disialylated lipo-oligosachharides induce the GQ1b ganglioside antibodies associated with Miller-Fisher syndrome (MFS). ${ }^{14}$ However, the pathogenic mechanisms in acute inflammatory demyelinating polyneuropathy (AIDP) are less clear with both cellular $^{15} 1^{16}$ and humoral immune responses reported. ${ }^{17}{ }^{18}$ Seasonal variation in the inducing illness may also effect the burden of disease due to GBS. Patients with GBS are usually admitted to hospital and artificial ventilation may be required in up to $25 \%$ of patients. Death occurs in $3-10 \%$ of patients, and there is significant residual morbidity in survivors with up to $20 \%$ of patients unable to walk at 6 months. ${ }^{19}$ This may have implications for seasonal demands on neurology, neurointensive care and neurorehabilitation services.

We performed a systematic review to identify seasonality in incidence of GBS globally and which factors affect the presence of seasonality. We then determined seasonality within a retrospective cohort of admissions to a large UK teaching hospital in the UK, determined seasonality in GBS admissions, associated prodromal illness, and length of stay.

\section{METHODS}

Systematic review

PubMed and EMBASE were searched with the terms ("seasonal" OR "season" OR "winter" OR "summer" OR "autumn" OR "spring”) AND ("Guillain Barre" OR "Guillain-Barre" OR "GBS" OR "acute inflammatory demyelinating polyneuropathy" OR "acute motor axonal neuropathy" OR "acute neuropathy") between inception and 31 
January 2014. Any study reporting GBS incidence by month, season or by the relative incidence comparing winter and summer was included, taking season as defined by the reporting study or, where monthly data was reported, using the timeframes used in our cohort, but inverted for countries in the southern hemisphere.

\section{Oxfordshire cohort}

We performed a retrospective cohort study of all patients discharged from the John Radcliffe Hospital, Oxford, with a diagnostic code of 'Guillain-Barré syndrome' between January 2001 and December 2012. Medical notes, correspondence and neurophysiological records were manually reviewed to confirm accuracy of coding for GBS, and identify admission and discharge date, plus any reported prodromal illness. Supportive neurophysiological studies were required for inclusion, to limit inclusion of inappropriate cases defined purely on clinical grounds that could reduce the study's sensitivity for any seasonal variation. Subtype of GBS was defined on the basis of the original report of clinical neurophysiological studies as AIDP, AMAN, acute motor sensory axonal neuropathy (AMSAN) or MFS (also defined by clinical features).

Admissions were divided into four seasons of 3 months (winter: January-March, spring: April-June, summer: JulySeptember, autumn: October-December). Seasonal variation was determined by a $\chi^{2}$ test (vs a uniform distribution) and as the incidence rate ratio (IRR) for winter versus summer, assuming a constant population size. Frequencies of prodromal infections in winter versus summer were compared by $\chi^{2}$ testing.

\section{Meta-analysis}

Meta-analyses of seasonal variation were performed on the natural logarithm of IRRs, using fixed and random effects meta-analysis weighted by the inverse variance. We calculated IRRs for each season versus the average of the other seasons,

Table 1 Characteristics of patients admitted with confirmed Guillain-Barré syndrome (GBS) to the John Radcliffe Hospital, Oxford, 2001-2012, according to GBS subtype. Mean (SD) values are given for continuous variables and $n(\%)$ for discrete variables

\begin{tabular}{lllll}
\hline \multirow{5}{*}{ Characteristic } & \multicolumn{4}{l}{ Guillain-Barré syndrome subtype } \\
\cline { 2 - 5 } & AIDP & AMAN & AMSAN & Miller-Fisher \\
\hline Number & 119 & 10 & 6 & 5 \\
Age & $52(21)$ & $39(24)$ & $60(9.5)$ & $47(18)$ \\
Gender & & & & \\
$\quad$ Women & $43(36)$ & $2(20)$ & $4(67)$ & $2(40)$ \\
$\quad$ Men & $76(64)$ & $8(80)$ & $2(33)$ & $3(60)$ \\
Season & & & & \\
$\quad$ Winter & $38(32)$ & $4(40)$ & $3(50)$ & $3(60)$ \\
Spring & $30(25)$ & $2(20)$ & $2(33)$ & $0(0)$ \\
$\quad$ Summer & $21(18)$ & $2(20)$ & $1(17)$ & $1(20)$ \\
$\quad$ Autumn & $30(25)$ & $2(20)$ & $0(0)$ & $1(20)$ \\
Prodrome & & & & \\
Respiratory & $16(13)$ & $0(0)$ & $3(50)$ & $3(60)$ \\
Diarrhoea & $13(11)$ & $5(50)$ & $1(17)$ & $0(0)$ \\
$\quad$ Other & $4(3)$ & $1(10)$ & $0(0)$ & $0(0)$ \\
$\quad$ Unknown & $86(72)$ & $4(40)$ & $2(33)$ & $2(40)$ \\
Length of Stay & $21(22)$ & $30(36)$ & $47(52)$ & $8(6)$ \\
\hline AMAN, acute motor axonal neuropathy; AMSAN, acute motor sensory axonal \\
neuropathy.
\end{tabular}

and calculated IRRs for winter versus summer and spring versus autumn, for the whole population and stratified by prodromal illness and subtype of GBS. Heterogeneity was determined by Cochrane's $Q$ and the $\mathrm{I}^{2}$ statistic, with publication bias assessed by funnel plots. Determinants of heterogeneity for the natural logarithm of the IRR between studies were also identified by general linear models, weighted by the inverse variance, assessing publication date, geographical region (westernised economies, Far East, Middle East, Indian subcontinent, Latin America), age, gender, frequency of flu-like prodrome, frequency of diarrhoeal prodrome or known C. jejuni infection and frequency of AIDP or AMAN subtype. General linear modelling was also performed after multiple imputations (SPSS) to generate five imputed data sets, given the high frequency of at least one unreported independent variable in published studies.

All analyses were performed in Microsoft Excel 2010 or IBM SPSS V.20.

\section{RESULTS}

Of 413 separate reports identified by the primary search and additional references, 129 potentially viable abstracts were reviewed, with 87 eligible papers reviewed in full. Of these, 45 studies reported 10698 patients presenting with GBS by month or season (43 any form of GBS, 8 reporting for AIDP and 8 for AMAN), (see online supplementary e-table 1 ).

\section{Oxfordshire cohort}

Of 212 hospital episodes coded, GBS, there were 140 cases of confirmed, incident GBS, comprising 119 AIDP, 10 AMAN, 6 AMSAN and 5 MFS. Across all subtypes, the mean age was 51 $(\mathrm{SD}=21$, range 2-87), with 89 (64\%) men (see table 1). Nerve conduction studies, consistent with GBS, were recorded for 136 patients, with the remaining 4 patients being diagnosed solely on clinical grounds with presentations consistent with MFS. Across all patients, the median length of hospital stay was 14 days (IQR 6-28, range 0-135 days).

There was seasonal variation in the rate of admission for GBS (see figure $1, \mathrm{p}<0.037$ ), with significantly more patients admitted in winter versus summer (IRR 1.92, 1.18-3.11, $\mathrm{p}=0.004$ ), with no difference in rates of admission in autumn versus spring (IRR $0.97,0.60-1.57, \mathrm{p}=0.55)$. There was significant winter versus summer variation for AIDP (IRR 1.81, 1.06-3.08, $\mathrm{p}=0.015$ ), but although there were also more admissions with other subtypes of

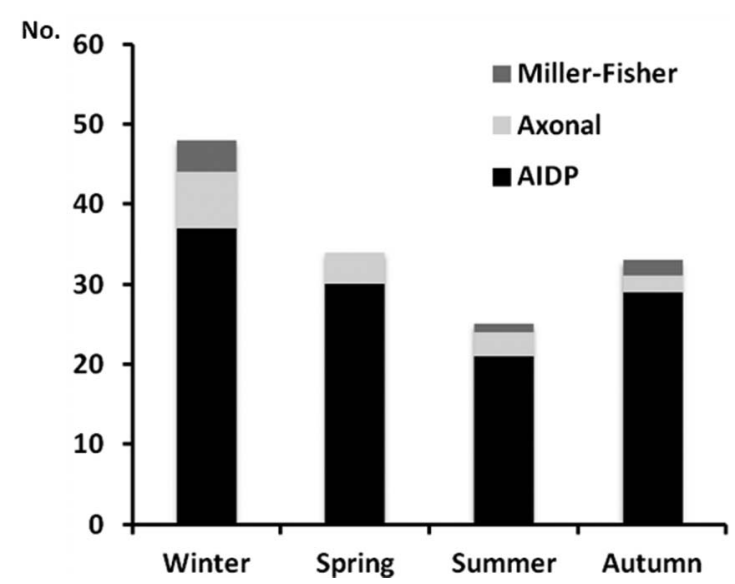

Figure 1 Seasonal variation in incidence of Guillain-Barré syndrome in a single tertiary referral centre in Oxford, 2001-2012, stratified by subtype. AIDP, acute inflammatory demyelinating polyneuropathy. 
GBS in the winter compared to other months there were too few patients to draw reliable conclusions (table 1). The mean length of stay was non-significantly shorter in the winter months (mean 15 days) versus summer (mean 21 days, $\mathrm{p}=0.08$ ). There was no significant seasonal variation in total bed occupancy (winter 736 days; spring 845 days; summer 521 days; autumn 972 days). The higher frequency of admissions in the winter was associated with a higher incidence of patients reporting a flu-like or respiratory prodromal illness in winter versus summer (11 vs 2, $\mathrm{p}=0.012)$, predominantly due to differences in frequency of AIDP preceded by a flu-like illness (7 vs $2, p=0.096$ ). There were insufficient cases of AMAN, AMSAN and MFS to reliably determine if there was an association between prodromal illness and these GBS subtypes, but all cases of AMAN in whom a prodromal illness was identified were subsequent to a diarrhoeal illness.

\section{Meta-analysis}

Across all studies, the incidence of GBS was greatest in the winter months, compared to the other three seasons, with both fixed and random effects meta-analysis (see e-figure 1). When comparing the winter and summer periods, a cluster of three studies in Northern China represented extreme outliers with a very high summer incidence of GBS, distorting the analysis such that there was a $12 \%$ greater incidence of GBS in the winter across all studies by fixed effects analysis, but no difference on random effects meta-analysis. However, these studies were marked outliers compared to other studies as evident on the funnel plots (see e-figure 2). Excluding these studies resulted in a significant $14 \%$ increased incidence of GBS in winter compared to the summer months, on both fixed and random effects analyses (figure 2), with no evidence of publication bias or other biases affecting the distribution of results (see e-figure 2). There was a small increase in incidence of GBS in autumn compared to spring but there was high heterogeneity, and this was not significant on random effects meta-analysis (see e-figure 3 ).

There was a significant heterogeneity between studies $\left(\mathrm{I}^{2}=78 \%, \mathrm{p}<0.0001\right)$, partly explained by its geographical location (figure 3), with a $28 \%$ increase in the winter incidence of GBS in Western countries, a $12 \%$ increase in winter in the Middle East and a 20\% increased risk in the Far East (after exclusion of the Northern China cluster). However, there was a $14 \%$ reduction in incidence in winter in the Indian subcontinent and a $25 \%$ decreased winter risk in limited studies from Latin America, although these results did not reach significance, limiting the reliability of any conclusion that there is no seasonal variation in these regions. This lack of seasonality in GBS was partly explained by the reversed (fixed-effects) or absent (random-effects) seasonality in tropical rainy climates compared to temperate climates, with no significant difference between temperate and tropical arid regions (see e-figure 4). Similarly, there was a greater predominance of GBS in patients with a respiratory prodromal illness in winter compared to patients with a diarrhoeal prodrome or C. jejuni positivity, and smaller, non-significantly greater winter predominance in patients with AIDP compared to axonal forms of GBS (figure 4).

Geographical region was a significant predictor of heterogeneity in winter versus summer IRR in general linear models

\begin{tabular}{|c|c|c|c|c|c|c|c|c|}
\hline Study & Events & IRR & Cilow & Cihigh & $\mathrm{p}$-value & p-het & & \\
\hline Ramirez-Zamora (2010) & 223 & 0.36 & 0.27 & 0.48 & $<0.0001$ & & — & \\
\hline Dowling (1977) & 85 & 0.57 & 0.37 & 0.89 & 0.0138 & & & \\
\hline Sriganesh (2013) & 68 & 0.70 & 0.43 & 1.13 & 0.15 & & - & t \\
\hline Nachamkin (2007) & 44 & 0.76 & 0.42 & 1.38 & 0.37 & & & 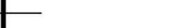 \\
\hline Dominguez-Moreno (2014) & 237 & 0.77 & 0.59 & 0.99 & 0.0447 & & $\longrightarrow$ & \\
\hline Kalita (2014) & 157 & 0.80 & 0.59 & 1.10 & 0.18 & & & - \\
\hline Esteghamati (2008) & 177 & 0.86 & 0.64 & 1.16 & 0.33 & & $\bullet$ & 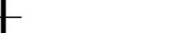 \\
\hline Chroni (2004) & 53 & 0.89 & 0.52 & 1.53 & 0.68 & & 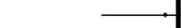 & \\
\hline Jiang (1997) & 864 & 0.91 & 0.80 & 1.04 & 0.17 & & & \\
\hline van konigsweld (2000) & 197 & 0.93 & 0.70 & 1.23 & 0.62 & & & - \\
\hline Paradiso (1999) & 39 & 0.95 & 0.51 & 1.78 & 0.87 & & & \\
\hline Chen (2013) & 188 & 1.00 & 0.75 & 1.33 & 0.99 & & & 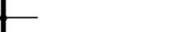 \\
\hline Yuan (2000) & 6 & 1.00 & 0.20 & 4.95 & 1 & & & \\
\hline Barzegar (2007) & 79 & 1.08 & 0.69 & 1.68 & 0.74 & & & \\
\hline Lyu (1997) & 56 & 1.15 & 0.68 & 1.95 & 0.59 & & & \\
\hline Govoni (1999) & 35 & 1.19 & 0.61 & 2.31 & 0.61 & & & \\
\hline Sivadon-Tardy (2006) & 126 & 1.19 & 0.84 & 1.69 & 0.33 & & & \\
\hline Sankhyan (2014) & 22 & 1.20 & 0.52 & 2.78 & 0.67 & & & \\
\hline Church Potter (2003) & 251 & 1.20 & 0.94 & 1.54 & 0.15 & & & $\cdot$ \\
\hline Stowe (2009) & 4250 & 1.23 & 1.16 & 1.31 & $<0.0001$ & & & 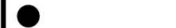 \\
\hline Jasem (2013) & 1289 & 1.25 & 1.12 & 1.39 & $<0.0001$ & & & \\
\hline Molinero (2003) & 220 & 1.27 & 0.97 & 1.65 & $<0.0001$ & & & \\
\hline Markoula (2007) & 16 & 1.29 & 0.48 & 3.45 & 0.62 & & & \\
\hline Tang (2011) & 133 & 1.29 & 0.92 & 1.82 & 0.14 & & & \\
\hline Baxter (2013) & 224 & 1.33 & 1.02 & 1.74 & 0.0331 & & & \\
\hline Bahou (1996) & 24 & 1.40 & 0.62 & 3.15 & 0.42 & & & \\
\hline Cuadrado (2005) & 57 & 1.48 & 0.87 & 2.51 & 0.15 & & & \\
\hline Deceunink (2008) & 10 & 1.50 & 0.42 & 5.32 & 0.53 & & & \\
\hline Blum (2013) & 164 & 1.52 & 1.11 & 2.08 & 0.0084 & & & \\
\hline Sharma (2011) & 23 & 1.56 & 0.67 & 3.59 & 0.3 & & & \\
\hline Cuadrado (2002) & 181 & 1.59 & 1.18 & 2.14 & 0.0025 & & & \\
\hline Hao (1998) & 91 & 1.60 & 1.05 & 2.44 & 0.0292 & & & \\
\hline de Pedro-Cuesta (1996) & 16 & 1.67 & 0.61 & 4.59 & 0.32 & & & \\
\hline Hung (2004) & 11 & 1.75 & 0.51 & 5.98 & 0.37 & & & \\
\hline Webb (2014) & 73 & 1.92 & 1.18 & 3.11 & 0.0082 & & & \\
\hline van Konigsveld (2001) & 33 & 2.00 & 0.97 & 4.12 & 0.06 & & & \\
\hline GBS study group (2000) & 46 & 2.29 & 1.22 & 4.28 & 0.0099 & & & \\
\hline Winner (1990) & 39 & 4.57 & 2.02 & 10.36 & 0.0003 & & & \\
\hline Ismail (1998) & 6 & 4.99 & 0.58 & 42.71 & 0.14 & & & \\
\hline \multirow[t]{2}{*}{ Boucquey (1991) } & 24 & 5.00 & 1.71 & 14.63 & 0.0033 & & & \\
\hline & & 5.00 & & & & & & \\
\hline Fixed Effects & 9837 & 1.15 & 1.10 & 1.19 & $<0.0001$ & I'2 $77.1 \%$ & & \\
\hline Random Effects & 9837 & 1.14 & 1.02 & 1.27 & 0.0201 & $<0.0001$ & & 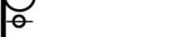 \\
\hline
\end{tabular}

Figure 2 Forest plot of the incidence rate ratio of Guillain-Barré syndrome (GBS) in studies comparing winter and summer. The cluster of reports from Northern China with a very high summer incidence of GBS are excluded. het, heterogeneity. 
Figure 3 Forest plot of the incidence rate ratio of Guillain-Barré syndrome in studies comparing winter and summer, stratified by region. het, heterogeneity.

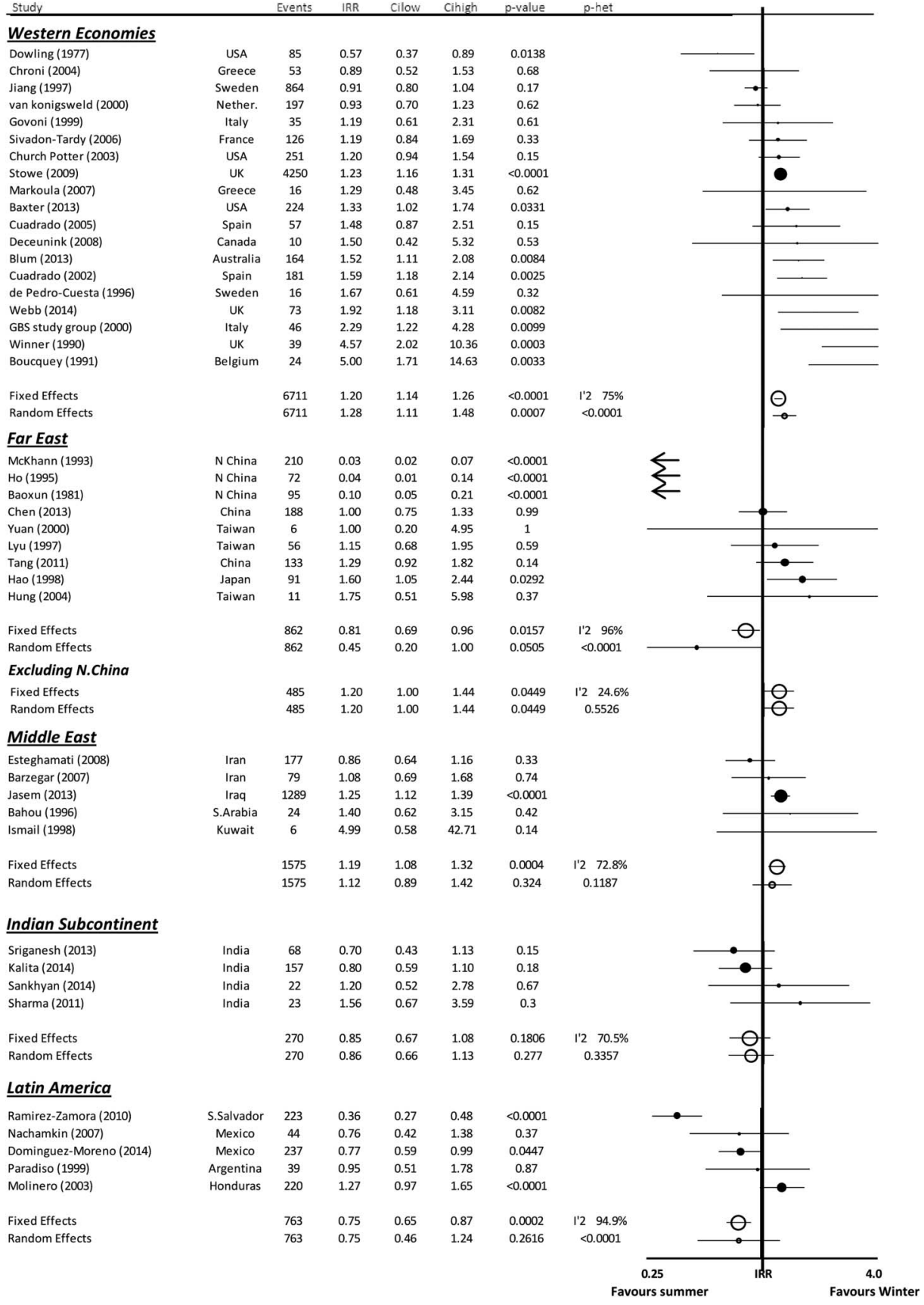

prodromal illness, but this was partially offset by a shorter length of stay in winter.

The seasonal variation in GBS in Oxford is consistent with previous publications from Oxfordshire ${ }^{120}$ and other studies in the $\mathrm{UK}^{3}$ which have suggested a winter predominance, but these did not consider the relationship with GBS subtype or prodromal illness. Our study is consistent with previous suggestions that seasonal variation within Western countries is due to the higher frequency of prodromal upper respiratory tract infections in winter, but there were too few cases in our cohort to reliably measure this. Diarrhoeal prodromal infections, particularly C. jejuni, are less common throughout the year in wealthier countries. ${ }^{21}$ In our study, there were too few patients with a diarrhoeal prodrome, confirmed C. jejuni infection or the AMAN subtype to identify any seasonal variations. However, it was noteworthy that all of the patients with AMAN had diarrhoeal symptoms (in those where a prodrome was recorded). 


\section{Study
Acute Inflammatory Demyelinating Neuropathy}

\section{Ho (1995)}

Lyu (1997)

Kalita (2014)

Paradiso (1999)

Blum (2013)

Webb (2014)

Nachamkin (2007)

Sankhyan (2014)

Fixed Effects

Random Effects

\section{$13 \quad 0.18$}

$31 \quad 0.82$

$117 \quad 0.89$

$27 \quad 1.08$

$77 \quad 1.41$

$58 \quad 1.76$

$14 \quad 1.80$

$4 \quad 3.00$

$341 \quad 1.12$

341

Cilow

Cihigh

p-value

p-het

$\begin{array}{ll}0.04 & 0.82\end{array}$

0.41

0.62

0.51

0.89

1.03
0.60

0.31

0.90

0.80
1.67

1.28

2.29

2.21

3.01

5.37

28.84

1.39

1.58

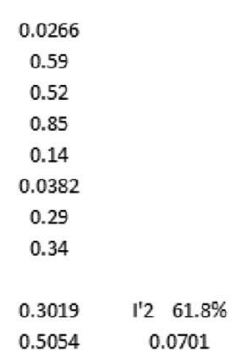

0.5054

\section{Acute Motor Axonal Neuropathy}

Ho (1995)
Nachamkin (2007)
Kalita (2014)
Paradiso (1999)
Lyu (1997)
Sankhyan (2014)
Blum (2013)
Webb (2014)
Fixed Effects
Random Effects

$\begin{array}{cccccc}59 & 0.02 & 0.00 & 0.12 & <.0001 & \\ 30 & 0.50 & 0.23 & 1.07 & 0.07 & \\ 39 & 0.63 & 0.33 & 1.19 & 0.15 & \\ 12 & 0.71 & 0.23 & 2.25 & 0.57 & \\ 4 & 1.00 & 0.14 & 7.10 & 1 & \\ 18 & 1.00 & 0.40 & 2.52 & 1 & \\ 87 & 1.64 & 1.06 & 2.52 & 0.0258 & \\ 10 & 2.33 & 0.60 & 9.02 & 0.22 & \\ & & & & & \\ 259 & 0.96 & 0.72 & 1.27 & 0.7653 & I^{\prime} 282.2 \% \\ 259 & 0.72 & 0.37 & 1.39 & 0.3245 & 0.0002\end{array}$

\section{GBS after respiratory infection}

Green (2001)
Sankhyan (2014)
Cuadrado (2002)
Cuadrada (2005)
Webb (2014)
Fixed Effects
Random Effects

46
65
59
25
13
208
208

1.56
2.42
4.90
5.25
5.50

2.76
3.06

0.86
1.42
2.48
1.80
1.22

2.81

4.13

9.67

15.29

24.81

0.14

0.0012

$<0.0001$

0.0024
0.0266

GBS after diarrhoea or c.jejuni infection

Cuadrado (2005)
Cuadrado (2002)
Takahashi (2005)
Green (2001)
Webb (2014)
Sharma (2011)
Fixed Effects
Random Effects

$\begin{array}{ccc}6 & 0.20 & 0.0 \\ 22 & 0.47 & 0.15 \\ 70 & 0.89 & 0.5 \\ 45 & 1.65 & 0.9 \\ 10 & 2.33 & 0.6 \\ 10 & 4.00 & 0.89 \\ & & \\ 163 & 1.06 \\ 163 & 1.10\end{array}$

$\begin{array}{lcc}0.02 & 1.71 & 0.14 \\ 0.19 & 1.14 & 0.1 \\ 0.56 & 1.43 & 0.63 \\ 0.90 & 3.01 & 0.1 \\ 0.60 & 9.02 & 0.22 \\ 0.85 & 18.84 & 0.08 \\ & & \\ 0.77 & 1.47 & 0.7064 \\ 0.60 & 2.00 & 0.7613\end{array}$

0.0002

$\begin{array}{cc}<0.0001 & \text { I' } 2 \quad 77.2 \% \\ <0.0001 & 0.0673\end{array}$
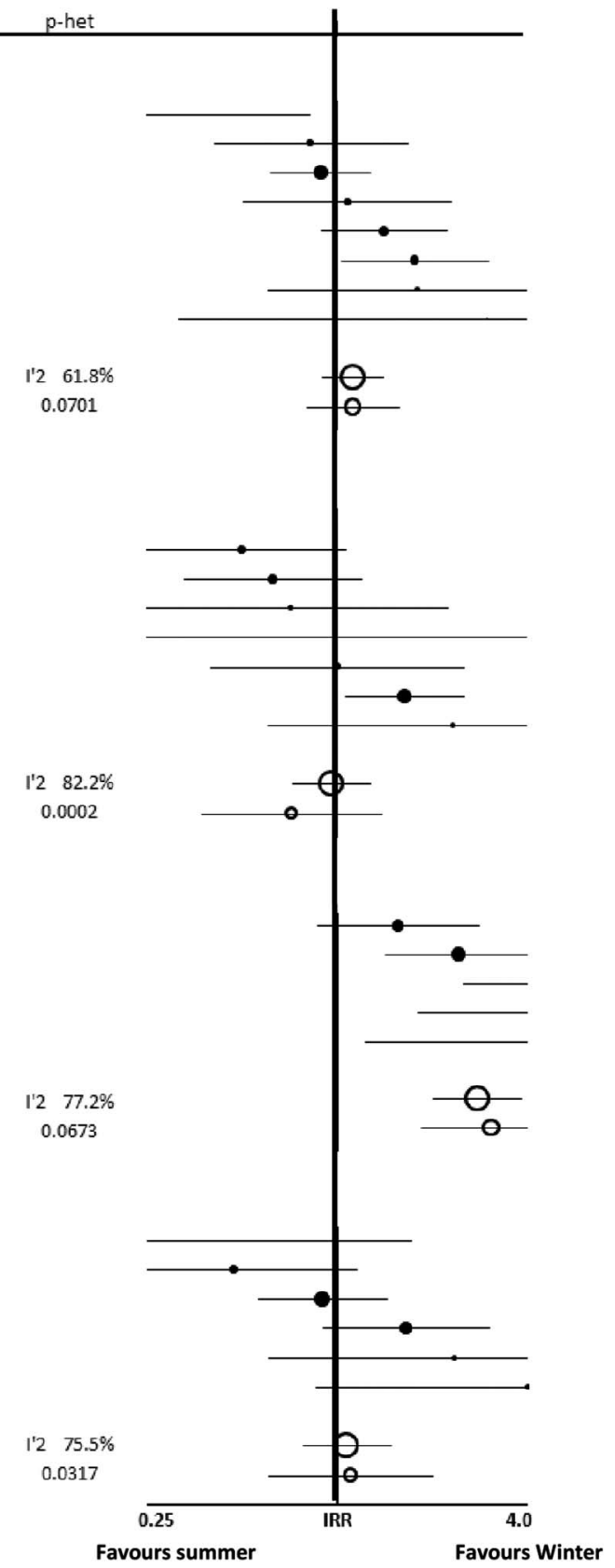

Figure 4 Forest plot of the incidence rate ratio of Guillain-Barré syndrome in studies comparing winter and summer, stratified by prodromal illness and electrophysiological subtype. het, heterogeneity.

There was no significant seasonal variation in bed occupancy in our cohort, despite the significant seasonal variation in GBS incidence. This may partly be explained by the non-significantly shorter mean length of stay in the winter months, which may reflect systematic differences in hospital care in the winter months, when a greater pressure on limited beds encourages earlier discharge from hospital. However, there may be some impact of a longer disease course with AMAN, which is relatively more frequent in summer months. ${ }^{19}$ However, we were not able to look for potentially adverse longer term patient outcomes in relation to this nor determine whether there was greater vigilance for GBS in patients with recent flu-like symptoms in the winter, falsely increasing the detected incidence of GBS.

Although there was seasonal variation in GBS incidence across the globe, both on average and in individual studies, there was significant heterogeneity between studies, part of which was explained by geographical region. Some of the variation in seasonal incidence may reflect the differing nature of seasons by latitude, with less significant differences between winter and summer in tropical regions, and potentially greater influence of other weather patterns such as the rainy season. There were also regions of contradictory seasonal variation in GBS incidence, in particular the high summer incidence of AMAN in Northern China, differing significantly from adjacent regions in the Far East, which showed winter predominance. This may reflect local variation in specific prodromal infections in rural communities. There were also broader regional differences in seasonal variation in GBS, with high winter predominance in Western countries, the Middle East and Far East, but summer predominance in the Indian subcontinent and Latin America. Again, this may reflect differences in the incidence of prodromal infections related to the season of the year, but may 
be related to local economic factors such as sanitation, ethnic differences in genetic susceptibility to GBS or the development of immune tolerance subsequent to previous viral infections. However, the data available does not allow us to discriminate between these factors.

Seasonal variation in GBS is particularly important for our understanding of the likely pathogenesis of the disease. This may have public health implications in the future for identifying prodromal infections that could be prevented to reduce the incidence of GBS in specific regions. It also has some potential implications for provision of tertiary neurological services, particularly in regions with a high incidence of summer GBS due to AMAN, which can result in longer admissions and greater bed occupancy, particularly in neurointensive care.

There are several limitations to this study. First, although there were a large number of studies reporting the seasonal incidence of GBS, these were primarily retrospective studies based on notes review (see online supplementary data) including our cohort, with few prospective studies and with significant differences in methodology between studies. This may contribute to ascertainment or under-reporting error, as suggested by the relatively high number of patients without a known prodrome in our cohort. Second, relatively few studies reported seasonal variation according to prodromal illness or GBS subtype, limiting the power of these analyses to identify the cause of underlying heterogeneity between studies. This significant heterogeneity was partly explained by geographical region and prodromal illness, but there were relatively few studies within the specific regions with low winter predominance of GBS (Latin America and the Indian subcontinent), resulting in only non-significant trends to lower winter predominance. Third, in our study, C. jejuni serology and antiganglioside antibodies were not systematically performed, and therefore these were not addressed in our cohort. Fourth, comparisons between AIDP and axonal GBS were hampered by the small numbers of these variants in our study and the limited number of publications reporting seasonal incidence by GBS subtype. Fifth, the definition of a season varied between studies, with other studies reporting the incidence of GBS by calendar month. Our definition of season was therefore chosen as the best compromise to allow synthesis between studies, rather than using an absolute meteorological definition. Finally, the majority of patients in our study had only one set of nerve conduction studies performed as this was the standard clinical practice at the time. It is therefore possible that some cases will have been misclassified as AIDP on electrophysiological grounds, given the recent appreciation that reversible conduction failure mimicking demyelination can be seen in pathologically axonal cases. ${ }^{22}$

In conclusion, there is significant seasonal variation in incidence of GBS in Oxfordshire and published studies with a winter predominance in most, but not all, geographical regions. This raises the possibility that targeted GBS education of primary care physicians, front-line acute hospital staff and resource managers in high-frequency periods might help to reduce adverse outcomes among the seasonal excess of GBS admissions. Furthermore, identifying the key immunological triggers by geographical region remains an important public-health challenge and offers a potential opportunity to intervene at the population level to reduce the mortality and morbidity associated with GBS, as indicated by the success of a New Zealand initiative to reduce C. jejuni contamination in poultry. ${ }^{23}$
Contributors AJSW designed and conducted the study, performed the analysis and drafted the manuscript. SAEB and RW collected and analysed data and contributed to the manuscript. SR contributed to analysis of data and editing of the manuscript. MRT conceived, designed and supervised the study, contributed to the analysis and edited the manuscript.

\section{Competing interests None.}

Ethics approval The cohort study was performed as an internal audit of hospital admissions at the John Radcliffe Hospital, approved by the Oxford University Hospitals NHS Trust.

Provenance and peer review Not commissioned; externally peer reviewed.

Data sharing statement MRT had full access to all the data in the study and takes responsibility for the integrity of the data and the accuracy of the data analysis.

\section{REFERENCES}

1 Winner SJ, Evans JG. Age-specific incidence of Guillain-Barré syndrome in Oxfordshire. Q J Med 1990;77:1297-304.

2 Boucquey $D$, Sindic CJ, Lamy $M$, et al. Clinical and serological studies in a series of 45 patients with Guillain-Barré syndrome. J Neurol Sci 1991;104:56-63.

3 Stowe J, Andrews N, Wise L, et al. Investigation of the temporal association of Guillain-Barre syndrome with influenza vaccine and influenza like illness using the United Kingdom General Practice Research Database. Am J Epidemiol 2009;169:382-8.

4 Chen Y, Ma F, Zhang J, et al. Population incidence of Guillain-Barré syndrome in parts of China: three large populations in Jiangsu province, 2008-2010. Eur J Neurol 2014;21:124-9.

5 Jiang $\mathrm{GX}$, Cheng Q, Link $\mathrm{H}$, et al. Epidemiological features of Guillain-Barré syndrome in Sweden, 1978-93. J Neurol Neurosurg Psychiatry 1997;62:447-53.

6 Ramírez-Zamora M, Burgos-Ganuza CR, Alas-Valle DA, et al. Guillain-Barre syndrome in the paediatric age: epidemiological, clinical and therapeutic profile in a hospital in El Salvador. Rev Neurol 2009;48:292-6.

7 Dowling PC, Menonna JP, Cook SD. Guillain-Barré syndrome in Greater New York-New Jersey. JAMA 1977;238:317-18.

8 Hughes RA, Rees JH. Clinical and epidemiologic features of Guillain-Barré syndrome. J Infect Dis 1997;176(Suppl 2):S92-8.

9 Ho TW, Mishu B, Li CY, et al. Guillain-Barré syndrome in Northern China. Relationship to Campylobacter jejuni infection and anti-glycolipid antibodies. Brain 1995; 118:597-605.

10 Baoxun Z, Yinchang Y, Huifen $\mathrm{H}$, et al. Acute polyradiculitis (Guillain-Barré syndrome): an epidemiological study of 156 cases observed in Beijing. Ann Neurol 1981;9:S146-8.

11 McKhann GM, Cornblath DR, Griffin JW, et al. Acute motor axonal neuropathy: a frequent cause of acute flaccid paralysis in China. Ann Neurol 1993;33:333-42.

12 Jacobs BC, Rothbarth PH, van der Meché FG, et al. The spectrum of antecedent infections in Guillain-Barré syndrome: a case-control study. Neurology 1998;51:1110-15.

13 Aspinall GO, Fujimoto S, McDonald AG, et al. Lipopolysaccharides from Campylobacter jejuni associated with Guillain-Barré syndrome patients mimic human gangliosides in structure. Infect Immun 1994;62:2122-5.

14 Koga M, Takahashi M, Masuda M, et al. Campylobacter gene polymorphism as a determinant of clinical features of Guillain-Barre syndrome. Neurology 2005:65:1376-81.

15 Massaro ME, Rodriguez EC, Pociecha J, et al. Nerve biopsy in children with severe Guillain-Barré syndrome and inexcitable motor nerves. Neurology 1998;51:394-8.

16 Griffin JW, Li CY, Ho TW, et al. Guillain-Barré syndrome in northern China. The spectrum of neuropathological changes in clinically defined cases. Brain 1995; 118:577-95.

17 Haymaker WE, Kernohan JW. The Landry-Guillain-Barre syndrome; a clinicopathologic report of 50 fatal cases and a critique of the literature. Med Baltim 1949;28:59-141.

18 Rinaldi S, Brennan KM, Kalna G, et al. Antibodies to heteromeric glycolipid complexes in Guillain-Barré syndrome. PLOS ONE 2013;8:e82337.

19 van Doorn PA. Diagnosis, treatment and prognosis of Guillain-Barré syndrome (GBS). Presse Med 2013;42:e193-201.

20 Winner SJ, Grimley-Evans J. Guillain-Barre syndrome in Oxfordshire: Clinical features in relation to age. Age Ageing 1993;22:164-70.

21 McCarthy ND, Gillespie IA, Lawson AJ, et al. Molecular epidemiology of human Campylobacter Jejuni shows association between seasonal and international patterns of disease. Epidemiol Infect 2012;140:2247-55.

22 Uncini A, Kuwabara S. Electrodiagnostic criteria for Guillain-Barrè syndrome: a critical revision and the need for an update. Clin Neurophysiol 2012;123:1487-95.

23 Baker MG, Kvalsvig A, Zhang J, et al. Declining Guillain-Barré syndrome after campylobacteriosis control, New Zealand, 1988-2010. Emerg Infect Dis 2012;18:226-33. 